\title{
Windowless Open Photoacoustic Helmholtz Cell
}

\begin{abstract}
T. STARECKI
Institute of Electronic Systems, Warsaw University of Technology

Nowowiejska 15/19, 00-665 Warsaw, Poland

A phenomenon which is usually considered as the main disadvantage of photoacoustic Helmholtz cells is photoacoustic background signal resulting from the light absorption by the cell windows. Although there are methods which reduce this background signal, e.g. light wavelength modulation or time domain filtering of the photoacoustic response from the sample, the methods are limited to particular applications, and none of them would completely remove the mentioned background signal. The paper presents design of a windowless open photoacoustic Helmholtz cell, and preliminary results of frequency response measurements of such a cell. Removal of the windows eliminated the main source of the photoacoustic background signal. Additionally, the proposed design allows for unrestricted or forced flow of the fluid filling the cell. As a result, the cell can be easily adapted for continuous, real-time photoacoustic measurements. Under proper selection of the cell dimensions, Q-factors of such open photoacoustic Helmholtz resonators are comparable to the Q-factors of corresponding closed Helmholtz cells.
\end{abstract}

PACS numbers: 43.38.Zp, 43.58.Kr

\section{Introduction}

Design of a photoacoustic cell depends strongly on its applications. Another important factor which must be taken into account is amplitude dependence of the photoacoustic signal [1]:

$$
A \propto \frac{P}{f V} Q,
$$

where $P$ - power of the light irradiating the sample, $f$ - light modulation frequency, $V-$ volume of the cell, $Q-$ quality factor of the cell (if the light is modulated at the resonance frequency of the cell).

According to Eq. (1), sensitivity of the setup (which is a crucial parameter in many applications) can be increased in at least a few ways, also influencing photoacoustic cell design. 


\section{Photoacoustic Helmholtz resonator}

A cell design which is often used in photoacoustics, especially in investigation of solid samples, is the Helmholtz resonator. In the simplest case it is an acoustic structure consisting of two cavities connected with a duct (Fig. 1). Resonance frequency $f_{0}$ of such a structure can be approximated by [2]:

$$
f_{0} \approx \frac{v \varphi}{4 \pi} \sqrt{\frac{\pi}{l} \frac{V_{1}+V_{2}}{V_{1} V_{2}}}
$$

where $V_{1}, V_{2}$ - cavity volumes, $l$ - length of the duct, $\varphi$ - diameter of the duct, $v$ - sound velocity in the fluid filling the cell.

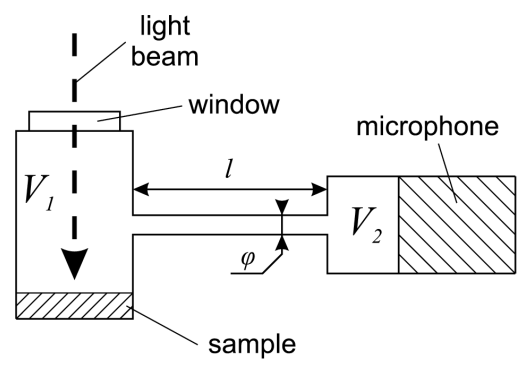

Fig. 1. Sketch of a photoacoustic Helmholtz cell.

It can be easily noticed from Eq. (2) that, on contrary to the cells that are based on standing wave resonances, resonance frequency of a Helmholtz resonator is not a simple linear function of its dimensions. This is due to a different mechanism of the resonance, which in the case of the Helmholtz cell results from a piston-like pressure stimulation in the sample cavity, leading to alternate pressure changes in the cavities and periodical flow of the fluid filling the cell between the cavities.

Even brief analysis shows several advantages of the Helmholtz resonator structure if applied in photoacoustic measurements. Due to two cavities it is possible to use $1 / 2^{\prime \prime}$ or even $1^{\prime \prime}$ microphones while keeping total volume of the cell small (a few $\mathrm{cm}^{3}$ or even less) [3, 4], which results in higher level of the output signal (see Eq. (1)). Simultaneously, although Q-factors of the Helmholtz resonators are not very high — usually a few to about 20 [5], they add additional gain to the setup sensitivity. Equation (2) shows that even at relatively small size it is possible to obtain quite low resonance frequencies, which may be used for further increase in the output signal. On the other hand, frequency of the resonance can be easily adjusted in a wide range by appropriate selection of the cell dimensions - e.g. diameter and length of the duct (hence, with virtually no influence on the total cell volume). Dual-cavity structure of the Helmholtz resonator allows for natural separation of the sample from the microphone, and prevents from interaction of the light with the microphone (which would produce a background photoacoustic 
signal resulting from absorption of the light by the microphone). It should be also mentioned that dual- or multi-cavity structures allow for easy shaping of the frequency response and also simplify implementation of differential setups, that may be used for reduction of acoustic noise as well as for additional increase in the measured signal (e.g. if the microphones are placed in the cavities in which the signal phase difference is $180^{\circ}$ ).

According to the mentioned advantages, the Helmholtz structure may seem to be a perfect solution for photoacoustic applications of any kind, while it is used mainly in the investigation of solids. What is usually considered as the main drawbacks of the Helmholtz resonator applied in photoacoustics are: background signal from absorption of the light by the cell windows and poor quality of the models based on acousto-electrical analogies, commonly used as design aids. It turns out, however, that although standard models do give sometimes huge errors (especially in calculation of Q-factors, even in modelling of relatively simple dual-cavity structures), it is possible to use loss-improved model [6], that gives much better agreement with the measurements, which finally makes window absorption the main disadvantage of photoacoustic Helmholtz cells.

\section{Multi-cavity and open Helmholtz resonators}

It should be noticed that the Helmholtz resonator may consist of more than two cavities. In comparison to dual-cavity circuits, such multi-cavity structures allow for more flexible forming of the frequency response of the cell. It also turns out that volume increase in the additional (third) cavity does not have to result in resonance damping (see e.g. Fig. 2) [7]. Moreover, there is no upper limit for the

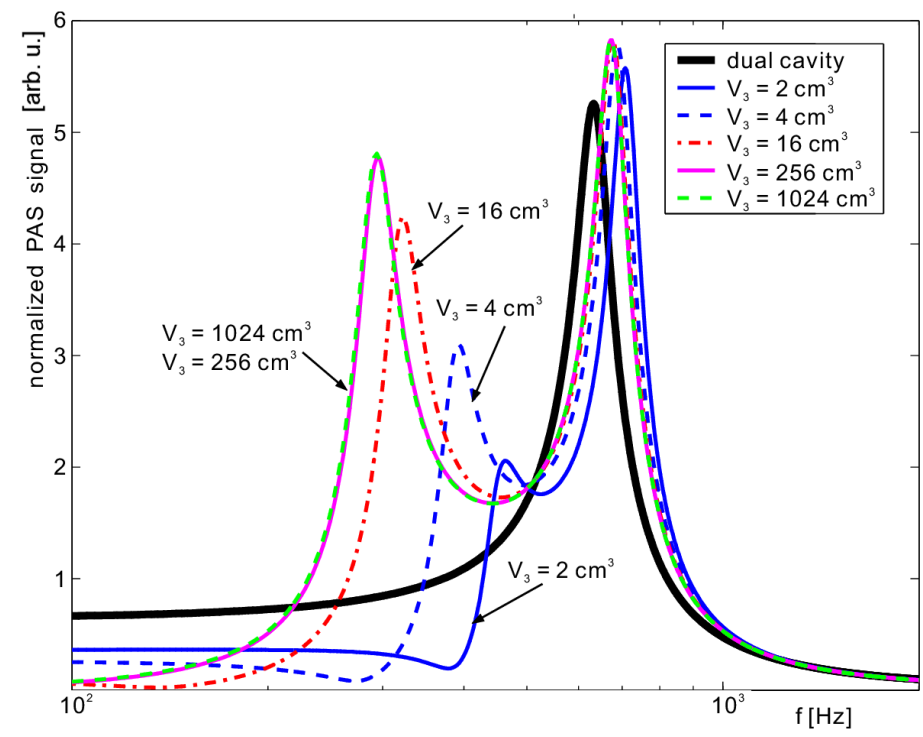

Fig. 2. Influence of the additional cavity volume on the frequency response of a triple-cavity photoacoustic Helmholtz cell. 
cavity volume, which means that in a multi-cavity open structure the exterior can be treated as a cavity. Such a feature is very valuable, because open cell allows for natural exchange of the fluid filling the cell with the exterior, which substantially simplifies design of the setup dedicated e.g. for water or air monitoring (no need for valves, pumps, etc.).

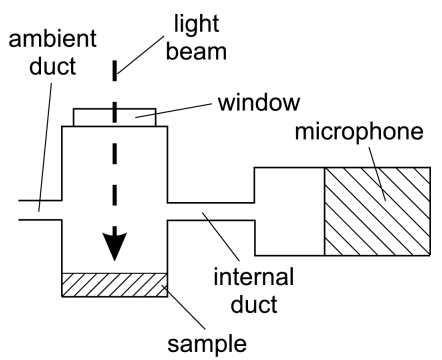

Fig. 3. A simple design of an open photoacoustic Helmholtz cell.

The simplest open structure with a Helmholtz resonance is a single cavity with a duct connecting the cavity with the exterior. Such a circuit is well known in acoustics [8], but it is not particularly useful in photoacoustic applications, as it does not have advantages of separation of the sample from the microphone, etc. For this reason a structure which can be considered as the simplest open Helmholtz resonator (of a practical value) in photoacoustics is a dual-cavity circuit connected with an additional duct with the exterior (e.g. Fig. 3).

\section{Windowless photoacoustic Helmholtz cell}

As already mentioned, background photoacoustic signal resulting from absorption of the light by the cell windows is usually considered as one of the main disadvantages of the Helmholtz structure. Windowless cell is probably the simplest solution which removes such a background signal. Taking into consideration, that it is possible to obtain an open Helmholtz resonator whose Q-factor will be comparable to the Q-factor of the corresponding closed structure, a windowless Helmholtz cell can be designed as in Fig. 4. It should be noticed that due to

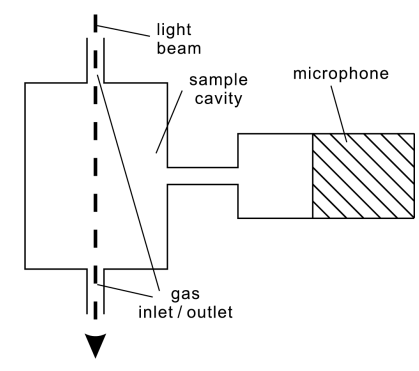

Fig. 4. Proposed design of an open windowless photoacoustic Helmholtz cell. 


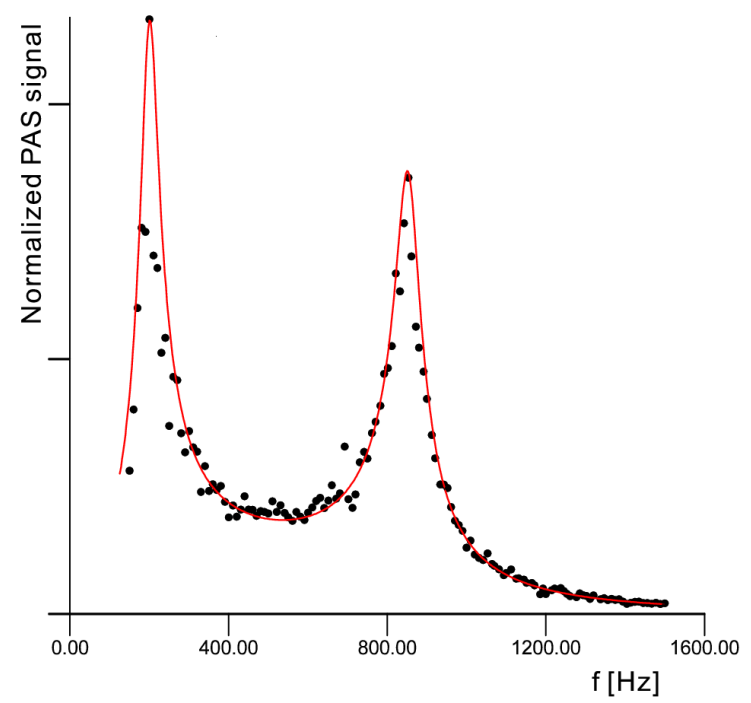

Fig. 5. Theoretical and measured frequency response of an open windowless Helmholtz resonator.

two openings (marked as gas inlet/outlet) such a cell can be conveniently used in forced flow applications. The main drawback of the presented solution is that it requires precise collimation and axial positioning of the light beam that must be passed through the inlet/outlet without interaction with the cell walls. However, this should not introduce big problems if the ducts have reasonable lengths and diameters, and a laser is used as the light source. Measured frequency response of such a cell (Fig. 5) shows very good agreement with theoretical values [6].

\section{Conclusions and remarks}

The proposed design of a windowless Helmholtz cell eliminates background photoacoustic signal from the windows absorption, thus cancelling or at least substantially reducing the main drawback of photoacoustic Helmholtz cells. The presented structure can be used in continuous flow experiments. Under proper selection of the ducts' dimensions Q-factor of the cell is comparable to the Q-factors of corresponding closed Helmholtz cells. Use of loss-correction model results in very good agreement between measurements and simulated frequency response of the cell. As a result, the Helmholtz structure can find new areas of applications in photoacoustics.

\section{References}

[1] Y.-H. Pao, Optoacoustic Spectroscopy and Detection, Academic Press, New York 1977.

[2] P.M. Morse, K.U. Ingard, Theoretical Acoustics, Princeton University Press, Princeton 1986. 
[3] E.M. Monahan Jr., A.W. Nolle, J. Appl. Phys. 48, 3519 (1977).

[4] D. Ducharme, A. Tessier, R.M. Leblanc, Rev. Sci. Instrum. 50, 1461 (1979).

[5] T. Starecki, A. Burd, S. Misiaszek, K. Opalska, M. Radtke, M. Ramotowski, in: Proc. II KKE, Kotobrzeg (Poland) 2003, Wydział Elektroniki Politechniki Koszalińskiej, Koszalin 2003, p. 653.

[6] T. Starecki, J. Acoust. Soc. Am. 122, 2118 (2007).

[7] T. Starecki, K. Opalska, A. Burd, S. Misiaszek, M. Ramotowski, Proc. SPIE 5948, 509 (2005).

[8] S.W. Rienstra, A. Hirschberg, An Introduction to Acoustics, extended and improved IWDE report 92-06, Eindhoven University of Technology, Eindhoven 2002. 